\title{
La fractura osteoporótica de cadera, una patología con abordaje multidisciplinar
}

\author{
Eduardo Montero Ruiz \\ Sección de Asistencia Compartida e Interconsultas. Servicio de Medicina Interna. \\ Hospital Universitario Príncipe de Asturias. Alcalá de Henares (Madrid)
}

La fractura de cadera de origen osteoporótico, tanto en sí misma como por las predicciones sobre su incidencia a corto-medio plazo, es un problema importante a nivel médico, social y económico. Médico, porque la mayor esperanza y calidad de vida de la población, unidas a la constante mejora en las técnicas quirúrgicas y anestésicas, hacen que reciban tratamiento quirúrgico por fractura de cadera pacientes de más edad y con una marcada comorbilidad, lo que dificulta enormemente su manejo por parte del traumatólogo, el cual solicita con frecuencia creciente la colaboración de diferentes especialistas. Además, debemos tener muy presente que el objetivo asistencial no es sólo conseguir los mejores resultados posibles del acto quirúrgico, también es reducir la mortalidad y conseguir la mejor recuperación funcional lo antes posible. Social, porque genera importantes trastornos tanto al paciente como a sus familiares, así como la necesidad de la intervención de fisioterapeutas, asistentes sociales y centros residenciales para su recuperación, generando una carga social con frecuencia difícil de soportar. Económico, por los gastos que genera a nivel médico y social.

Desde un punto de vista clínico, el que más nos compete a nosotros como internistas, a todas las dificultades anteriormente señaladas se añaden evidencias científicas crecientes que permiten mejorar los resultados obtenidos sobre diversos aspectos, como son la prevención y tratamiento del delirium perioperatorio, la restricción de las transfusiones sanguíneas o la profilaxis de complicaciones infecciosas como la infección de la prótesis articular, entre otros.
Todo ello aumenta más si cabe, la necesidad de implicar en la atención médica de estos enfermos a diversos especialistas médicos, sin olvidar el papel fundamental del personal de enfermería, fisioterapeutas y asistentes sociales. Un problema añadido es que, al tratarse de una patología que requiere un tratamiento urgente o urgente diferido, precisa de unos mecanismos de actuación bien establecidos, tanto a nivel asistencial como en la gestión hospitalaria, que interfieran lo menos posible en el funcionamiento normal del servicio de traumatología y la programación de quirófanos.

Esta situación ha inducido la puesta en marcha de diversas iniciativas para mejorar la asistencia y gestión de estos enfermos, siendo la fractura osteoporótica de cadera un claro ejemplo de una patología que requiere un abordaje multidisciplinar. Entre las soluciones adoptadas están el simple incremento de las interconsultas a servicios médicos, el establecimiento de mecanismos de interconsulta más o menos "automáticos" y diferentes nuevos métodos de colaboración entre internistas o geriatras y traumatólogos. Entre estos últimos está la creación de unidades de cadera con diferentes grados de implicación de los especialistas, la asistencia compartida o el ingreso de los pacientes con fractura de cadera en los servicios de medicina interna.

Todo lo comentado hasta ahora puede explicar que, desde que se publicó la primera guía clínica sobre fractura de cadera en $1997^{1}$, hayan aparecido muchas más, si bien la mayoría incompletas y con diferencias entre sí $i^{2}$. En general, las guías de práctica clínica dan recomen- 


\section{"debemos tener muy presente que el objetivo asistencial no es sólo conseguir los mejores resultados posibles del acto quirúrgico, también es reducir la mortalidad y conseguir la mejor recuperación funcional lo antes posible"}

daciones explícitas, que deben tener validez interna y externa, e intentan influir en la práctica clínica aportando estrategias para mejorar los resultados, disminuir la gran variabilidad clínica existente e incrementar la eficiencia de los hospitales, entre otras características propias. Su principal problema es el escaso seguimiento que suelen tener. Una forma muy útil y práctica de abordar este problema es mediante la implantación de vías clínicas que, a diferencia de las guías clínicas, son una forma de adaptar éstas a la práctica diaria real de un centro concreto. Describen los pasos que deben seguirse, la secuencia temporal o las responsabilidades de los diferentes profesionales que intervienen, entre otras características. Su existencia implica un compromiso real de ese centro y esos profesionales para mejorar la asistencia sanitaria a un grupo concreto de enfermos.

En este número de Galicia Clínica se publica la "Vía clínica para pacientes hospitalizados con fractura osteoporótica de cadera" realizada en el Hospital Universitario Lucus Augusti de Lugo $^{3}$. Nace con el aval de ser un centro con amplia experiencia en el abordaje multidisciplinar de la fractura osteoporótica de cadera, pionero al haber publicado la primera guía española sobre el tema ${ }^{4}$. La vía es muy completa, está bien estructurada y cuenta con la participación de todos los servicios y actores implicados en la atención a estos enfermos, incluida la dirección del hospital. Además, ofrece el nivel de evidencia en el que se fundamentan sus recomendaciones. En la primera parte, de manera concisa pero completa se describe la situación e implicaciones de la fractura osteoporótica de cadera, la metodología y objetivos de la vía y los diferentes procesos y subprocesos que la componen. Al final, se describen una serie de recomendaciones reunidas en tablas, lo que facilita su lectura y aplicación por cada miembro del equipo implicado. Ante la calidad de esta vía como punto de partida, será fácil en el futuro valorar su impacto sobre el proceso de la fractura osteoporótica de cadera y realizar las modificaciones a que diera lugar. Un dato que refrenda el sentido común y buen hacer de los autores, relacionado con un aspecto muy importante y que cada vez se ignora más, es que, como ellos mismos subrayan, las guías, vías y protocolos son una gran ayuda para el clínico, pero no sustituyen su valoración y juicio clínico.

Para finalizar, la publicación de esta vía clínica en una revista médica es una buena noticia por dos razones. La primera, porque pone de manifiesto la voluntad y el interés práctico por parte del Hospital Universitario Lucus Augusti y sus profesionales, en mejorar la atención a estos pacientes. La segunda, porque la difusión de vías clínicas es muy poco frecuente, a pesar de su gran utilidad potencial, y pueden facilitar a otros centros la posibilidad de adaptarlas a su entorno y necesidades.

\section{Bibliografía}

1. Scottish Intercollegiate Guidelines Network (SIGN). Management of elderly people with fractured hip. Edinburgh: SIGN; 1997.

2. Bardales Mas Y, González Montalvo Jl, Abizanda Soler P, Alarcón Alarcón MT. Guías clínicas de fractura de cadera. Comparación de sus principales recomendaciones. Rev Esp Geriatr Gerontol. 2012; 47: 220-27.

3. Monte-Secades R, Codesido-Vilar P, Pardo-Sobrino FJ, García-Monasterio El, Portero-Vázquez A, Garcia-Novio M, Fernández-Lamelo F. Vía clínica para pacientes hospitalizados con fractura osteoporótica de cadera. Galicia Clin 2016; 77 (2): 57-66

4. Monte Secades R, Rabuñal Rey R, Bal Alvaredo M, Guerrero Lombardía J. Guía clínica para la atención de los pacientes con fractura de cadera. Disponible en www. meiga.info/guias/FracturaCadera.asp. Accedido el 19 de abril de 2016. 